\title{
MODOS DE CONSUMO Y SOCIEDAD DEL RIESGO*
}

\author{
JAVIER CALLEJO GALLEGO \\ UNED. Madrid
}

\section{PALABRAS CLAVE ADICIONALES}

Riesgo,Decisión, Elección, Seguridad, Producción social del consumidor.

\section{ADDITIONAL KEYWORDS}

Risk, Decision, Choice, Security, Consumer Social Production.

RESUMEN. Este trabajo aborda la introducción del riesgo en el campo del consumo. Una introducción que tiene tanto dimensiones reales, como han mostrado distintas crisis de consumo, como conceptuales. El artículo ha optado por apoyar los cambios conceptuales en el consumo, con la inserción del riego, en trabajos empíricos de tres investigaciones. Antes, se han establecido tres niveles de relación entre consumo y riesgo: metamercantil, mercancías, seguridad y riesgos específicos de las mercancías. Tres niveles fuertemente articulados. El análisis de los discursos recogidos destaca cómo la reducción del primero de los niveles (metamercantil) conduce a una extensión de los otros dos. Así, se llega a una conclusión paradójica: aun cuando ha aumentado la percepción de riesgo en el consumo, este campo sigue concibiéndose como uno de los de más seguridad.

ABSTRACT. This work tackles the introduction of risk in the consumption field. An introduction with real - as several crisis of consumption have shown - and conceptual dimensions. The paper has decided to support conceptual changes in consumption, about the risk insertion in it, on empirical work from three researchs. Before, three levels of consumption and risk relations have been established: metagoods, security goods and specific risk of goods. Three strongly articulated levels. The analysis of collected discours emphasizes that the first level (metagood) reduction drives to a spread of the other two levels. So, a paradoxical conclusion is reached: the field of consumption keeps as a safe field, in spite of the fact that risk perception has grown in it.

\footnotetext{
* Quiero expresar mi agradecimiento a los comentarios que Cecilia Díaz y Cristóbal Gómez hicieron al texto original, quienes, por supuesto, no son responsables de las deficiencias que puedan contener estas páginas.
}

E-mail: mcallejo@poli.uned.es

\section{Revista Internacional de Sociología (RIS)}

Tercera Época, N 40 , Enero-Abril, 2005, pp.133-157. 
RIS

REVISTA INTERNACIONAL DE SOCIOLOGÍA

No 40, ENERO-ABRIL, 2005

JAVIER CALLEJO GALLEGO

\section{INTRODUCCIÓN}

El término riesgo se ha insertado en el centro del debate de las ciencias sociales en los últimos años. En el campo del consumo, podría decirse que ha irrumpido de manera especial de la mano de las casi periódicas crisis alimentarias experimentadas durante el último decenio en distintos puntos de la sociedad occidental. Aun cuando han afectado principalmente a un determinado tipo de consumo, como es el de alimentos, parece pertinente plantear la reflexión sobre el conjunto del campo del consumo. Así, se propone enfocar, con apoyo en investigaciones empíricas, las articulaciones que se establecen entre riesgo y consumo.

En su acepción más generalizada, el riesgo tiene que ver con el cálculo de probabilidades y con la anticipación de las consecuencias de la acción, en función de tal cálculo. Pero puede considerarse que ésta es sólo una de las concreciones que toma el riesgo en el campo del consumo, vinculado especialmente a unas mercancías específicas (seguros y productos semejantes). Es más, ahondando en sus lógicas respectivas, se observa que la lógica del riesgo y la del consumo se encuentran en la aceptación de los individuos como sujetos que eligen o deciden, de manera que tanto consumo como riesgo se convierten en ejercicios individuales de libertad.

\section{ELECCIÓN Y DECISIÓN}

El análisis de la relación entre consumo y riesgo ha sido reciente y ha sobrevenido de modo abrupto. Tal vez nos haya despertado de un sueño: el de la exclusión de la inseguridad del consumo. Mejor dicho, el sueño de la exclusión de la incertidumbre para el consumo, pues, situado en su contexto histórico, se produce seguridad para el consumo. La sociedad de consumo aparece como una excepción de seguridad en una historia dominada por la inseguridad. Es más, desde distintas perspectivas, se establece la relación entre riesgo y modernización capitalista, a la que cabe aceptar como la base de la sociedad de consumo: ya sea desde la crítica marxista, que señala la eterna inseguridad y la movilidad como rasgos distintivos de la época burguesa (Marx y Engels, 2002:42), ya sea desde la racionalización descriptiva, como la de Luhmann (1998), que une aumento de complejidad y de riesgo. Sin embargo, el consumo surge como una isla en un mar de riesgos intrínsecos a la modernización.

Riesgo y extensión del consumo aparecerían, así, como dos polos opuestos del proceso de modernización. Pero hay una vinculación entre consumo y riesgo: ambos conceptos parecen situarse en la elección. Elección entre bienes, en el caso del consumo; elección entre comportamientos, en el del riesgo. De hecho, lo que diferencia riesgo y peligro es, en principio, la incrustación de la elección en el primero. El riesgo se asume, se toma, se racionaliza en clave de evaluación y cálculo 
de probabilidades. El peligro está, se impone a los individuos. La definición del riesgo en el ámbito de las elecciones facilita su ubicación en el ámbito del consumo y, en definitiva, en la responsabilidad de los consumidores. Sin embargo, este paso hacia las responsabilidades y el sentido del supuesto elector es lo que lleva de las elecciones a las decisiones, que han de tomarse como la concreción de la acción de los sujetos en clave de riesgo, pues, como señala Ramos (1999:258), la decisión es fundamental en la semántica del riesgo'. Entre sociedad de consumo y riesgo existen las mismas cercanía y distancias que entre elección y decisión.

Parece oportuno traer la distinción que Giddens (1997) hace entre sociedad de elección y decisión. Asumiendo la extensión de la dominación de la segunda en el proceso de modernidad tardía, Giddens establece la decisión como una acción de poder y, sobre todo, como el poder del experto, si bien acentuando más el lugar del experto profesional, que el del experto práctico, del que obtiene su pericia y relación con un campo de actividad por la propia experiencia y no a través de procesos formales y títulos formales de reconocimiento que generan los profesionales. Pero aquí interesa resaltar tanto el paso de la elección a la decisión, en la medida en que aumenta la experiencia de los individuos en un campo, sea formalmente reconocida o no, como la decisión como una acción llena de dudas y sombras, precisamente porque se tiene experiencia en el campo de referencia concreto. Puede hablarse, así, de elecciones inocentes frente a decisiones comprometidas.

En la elección - del latín eligere: "sacar", "escoger"-hay una serie de alternativas en un marco de seguridad vital. En la decisión-del latín decidere: "cortar", "no vuelta atrás" - se acepta un sentido vital. En las decisiones no hay alternativas, el sujeto es elegido. La elección revela unas preferencias (Sen, 1982), mientras que la decisión descubre estrategias. La elección es formalmente racional ${ }^{2}$, accesible a la guía del cálculo. La decisión, consecuencia de un contexto pragmático de riesgo, se encuentra en la racionalidad material, ese concepto equívoco del que habla Weber (1979:64), que va más allá del cálculo, por encontrarse mediado por normas.

A pesar de la diferencia entre elección y decisión, que vincula el riesgo a la segunda, han dominado las aproximaciones entre sociedad de consumo y riesgo en clave de la asimilación del riesgo con la elección. Una definición del riesgo en clave de elecciones del consumidor evoca directamente el argumento de la soberanía del consumidor. La estructura de tal modelo sería semejante a la del modelo utilitarista del consumo: el consumidor se comporta con respecto a la mercancía en función de la utilidad esperada en las mismas. Con la introducción del riesgo

\footnotetext{
${ }^{1}$ Sobre la vinculación entre decisión y riesgo, véase especialmente Luhmann (1992).

${ }^{2}$ La acción puramente racional, en función de cálculos de probabilidades de éxito es un ideal, sólo al alcance de máquinas.
} 
se añadiría otro elemento, el de utilidad negativa. La teoría convencional quedaría simplemente amplificada, de tal manera que la función de utilidad integraría las probabilidades de esas utilidades negativas o peligros. Integraría el riesgo sin moverse un ápice de una soberanía del consumidor, que deja a éste la acción de elegir entre las alternativas ofertadas. El consumidor valoraría la mercancía en función de la expectativa de utilidad de la misma, su precio y, ahora, la percepción de probabilidad de utilidad negativa (riesgo). Así, desde tal perspectiva, el consumidor racional podría asumir altas probabilidades de riesgo en función de precios interesantes; compraría, rebajándolo del precio, riesgo. El problema técnico, que deriva directamente del error epistemológico que asigna elevadas posibilidades de elección al consumidor, se encuentra en la dificultad para estimar el riesgo percibido, aun cuando se han hecho algunos intentos (Viscursi y Evans, 1998).

La asimilación de riesgo con elección en el consumo, aleja el análisis sociológico del consumo de la producción ${ }^{3}$ y la posición de los sujetos en la producción. El riesgo queda encerrado en la elección de las mercancías. Es como si el riesgo fuera algo intrínseco a las mercancías. Habría mercancías con riesgo y mercancías seguras. El papel del consumidor se limitaría a la elección. Los consumidores harán elecciones en presencia de incertidumbre y riesgo (Jakus y Shaw, 2003), apoyadas en las percepciones del propio consumidor o en la mediación de comunicaciones expertas (asesores, profesionales, asociaciones) o interesadas (publicidad en sus distintas concreciones). El papel de las instituciones mediadoras, como el Estado, estaría casi limitado a ofrecer (u obligar a dar) información sobre los riesgos inscritos en algunas de estas mercancías: el tabaco perjudica la salud, o este producto tiene un $\mathrm{x}$ por ciento de manipulación genética. Se abre la sospecha de un proceso homólogo al que Marx describió en el "fetichismo de la mercancía", cuando critica a la economía política burguesa por fijar el valor de las mercancías en la escasez y la demanda. Ahora, se sitúa el valor riesgo como algo propio de la mercancía. Sin embargo, parece clave distanciarse de esta concepción y preguntarse también si esa especie de contravalor (el valor riesgo), al encontrarse precisamente en la percepción del consumidor, lo está por la posición de éste en el mundo, incluido el mundo de la producción. Claro está, al igual que hizo el análisis marxista, se está hablando de producción social de la mercancía y, por lo tanto, de producción social del riesgo. Entonces, son las condiciones de producción las que generan el riesgo y no las condiciones atribuidas a la mercancía.

\footnotetext{
${ }^{3}$ En el campo alimentario, el cambio desde la demanda de control de los procesos de transformación hacia los procesos de producción es sintomática en los informes realizados por las instituciones internacionales FAO, OMS y la Comisión Europea (Libro Blanco de la Seguridad Alimentaria), como un cambio de estrategia ante los relativos resultados de la anterior. Véase Neira González (2003).
} 


\section{PRODUCCIÓN SOCIAL Y CONSTRUCCIÓN SOCIAL DEL RIESGO}

Al menos antes de que Beck (2002) intentase zanjar la cuestión sobre si los riesgos percibidos pertenecen a la realidad o son meramente percepciones subjetivas, el debate tuvo su relevancia. Desde el constructivismo social, se genera la afirmación de que el evidente crecimiento de la sensación de riesgo es producto de una mayor sensibilidad hacia el mismo, dentro de una sociedad desarrollada en la seguridad y que ha hecho de ésta parte de la demanda. Se unirían así la sensación de riesgo como un producto del desarrollo de la sociedad y, por lo tanto, del propio progreso, y la demanda de seguridad como un interesante campo para la mercancía. Lo que se estaría realmente produciendo es más seguridad, ante una constantemente insatisfecha demanda de la misma. Es un análisis un tanto naïf, que, además de su arraigo, tiene también sus concreciones bastante más interesantes. En especial las situadas en el entorno de la denominada teoría cultural, donde, especialmente representada por la figura de Mary Douglas (1992, 1996 y 1998), las sociedades siempre habrían estado atravesadas por la sensación de riesgo, aunque de distinta manera entre sus componentes. Para ello, establece una tipología de cuatro posiciones, especialmente definidas por su relación con el dominio y con la autoridad, que, en lo que aquí respecta, se correspondería con cuatro percepciones distintas del riesgo. Establece cuatro estilos fundamentales de riesgo, presentados de tal manera que parece que, rechazando explícitamente explicaciones en clave psicológica, es cuestión de seleccionar un estilo de vida ${ }^{4}$.

Los realistas, por su lado, remarcan que, más allá de que una parte de la evolución dé la sensación de riesgo, existe un riesgo real: las personas han padecido enfermedades, incluso la muerte, derivadas de los efectos de algunas fuentes consideradas de riesgo, lo que puede ir desde centrales nucleares, a carne de las denominadas "vacas locas". Tan sano ejercicio de realismo puede quedarse corto si se limita a establecer la división entre comportamientos y mercancías seguros y comportamientos y mercancías inseguros, en función de sus consecuencias más o menos objetivas.

Parece fácil mediar en el debate entre si hay más sensación de riesgo porque hay más fuentes de riesgo, $\mathrm{o}$, al contrario, si hay más fuentes de riesgo porque hay mayor sensibilidad hacia el riesgo ${ }^{5}$. Pero, ¿de dónde surge tal aumento de la sensación y, a la vez, la realidad de riesgo? Una pregunta donde es pertinente

\footnotetext{
${ }^{4} \mathrm{La}$ inserción de los estilos de riesgo en estilos de vida en la teoría cultural es lo que posibilita la conexión de actitudes ante el riesgo y las preferencias políticas, resultado al que llegan Dake y Wildavsky (1990).

${ }^{5}$ Gil Calvo media en el debate poniéndolo en una concreción histórica: "lo que ha crecido con la globalización no es tanto el riesgo real como el conocimiento público del riesgo percibido" (Gil Calvo, 2003:38).
} 
RIS

REVISTA INTERNACIONAL DE SOCIOLOGIA

No 40, ENERO-ABRIL, 2005

JAVIER CALLEJO GALLEGO

descender a la relación concreta con la sociedad de consumo. Así, hay que destacar cómo se produjo seguridad para lanzar la sociedad de consumo: empleos estables, protección frente a las incertidumbres (salud, vejez), extensión del crédito (venta a plazos), etc, (Callejo, 2001). En definitiva, el lanzamiento de la sociedad de consumo se llevó a cabo a partir de la extensión de la ciudadanía (Alonso, 2000), a través del empleo y el salario, que supuso la institucionalización del denominado Estado del bienestar. La sociedad de consumo nace en la sociedad del trabajo. Durante algún tiempo, la seguridad en el empleo y los salarios soportó la estabilidad de la sociedad de consumo y, a la vez, ésta la seguridad de aquélla. El modo de acumulación fordista estaba regulado por normas de seguridad, una integración que aparece rota en nuestros días, mostrando el aumento del riesgo como su sombra.

\section{EL CONSUMO: VITAL SEGURIDAD}

Frente a la concepción de los constructivistas sociales, más centrada en la percepción, se propone aquí analizar el riesgo desde su producción social. Esto quiere decir que no habría estilos de riesgo, sino, a lo sumo, distintos modos de producción del riesgo que, en cada caso, generan distintos estilos. Para ello, se propone situar la producción de riesgo en la producción social. Esto significa, en principio, reducir el margen de tentación por el riesgo de los consumidores. $\mathrm{Al}$ menos, situar tal tentación como producida.

Como señala Baudrillard (1970), la sociedad de consumo se produce como ámbito "mágico" de seguridad. Es el mundo de la seguridad, algo que realza Galbraith (1985) al plantear que la única fuente de temor que queda en la constitución de la sociedad opulenta (la sociedad de consumo) es la del cobrador.

Tal acento inicial en la seguridad, con la creación de instituciones que velasen por la misma, se incrusta en el fomento del consumo, en la socialización del consumo (Aglietta, 1979). El fruto de la socialización previa en el ahorro y el esfuerzo hizo que la demanda de seguridad para el consumo apareciese, desde el principio, con unas dimensiones insaciables.

El consumidor siempre ha tendido a la seguridad, a aferrarse a la misma. El consumidor requiere estabilidad y predictividad. Ésta es buena parte del éxito del lanzamiento de la sociedad de consumo, que puede entenderse como un pacto de estabilidad, y de su desarrollo. En tal predictividad parece apoyarse la extensión de empresas de consumo de masas (Ritzer, 1999; Ritzer y Miles, 1998).

Hay que resaltar cómo tal búsqueda de seguridad en el consumo tiene mayor relevancia en campos del mismo en los que secularmente, antropológicamente podría decirse, ha existido una inseguridad y desconfianza latente, como es el caso de la alimentación (Fischler, 1995; Millán, 2000). En otros campos, dada la seguridad que se proyecta en el consumo, pueden llegar a convertirse 
en una especie de refugio en una sociedad del riesgo. En una sociedad donde la identidad de las personas aparece en constante amenaza, bajo turbulencias (Giddens, 1995), el consumo puede convertirse en ámbito en el que construir la identidad.

Buena parte de la sensación de seguridad de nuestras vidas se adquiere a través de un consumo en el que se nos sitúa como los depositarios de la decisión de compra, yendo más allá de la formal soberanía del consumidor. Así, no sólo hace falta seguridad para consumir sino que, también, el consumo es fuente de seguridad vital en la que nos constituimos. Es más, si en un principio el proceso se estableció desde la constitución de la seguridad del ciudadano hacia la consecución de la seguridad del consumidor, ésta se convierte en un refugio, lo que hace que aumente la demanda sobre ella, a medida que avanzan las áreas de incertidumbre en la constitución de la ciudadanía y de la identidad. Es en el consumo donde se compone la identidad, que ha dejado de obtenerse en la producción. Como dice Bauman (1999:51), estamos en una sociedad de consumidores que se contenta con estas identidades poco duraderas y reemplazables.

El comportamiento de consumo se encuentra vinculado al rechazo al riesgo, incluso en una sociedad donde todo parece presentarse bajo la dimensión del riesgo (Beck 1998; Luhmann 1998); circunstancia que no hay que confundir con la existencia de sectores de la sociedad que parecen inclinarse, en sus elecciones de consumo, por ciertas prácticas de riesgo, como son los llamados deportes de riesgo. Precisamente por denominarse deportes de riesgo, son los deportes con más acento sobre el control, realizados en un contexto de control del mismo ${ }^{6}$. Pero incluso el consumidor de estas prácticas exige, en mayor medida que en otros campos, que todos los productos y elementos adquiridos para su desarrollo estén en las mejores condiciones. El riesgo, en cuanto aumento del margen de incertidumbre, realmente empezaría cuando tales elementos, incluido el entrenamiento del practicante, dejasen de estar en las mejores condiciones.

La demanda de unos sectores sociales de consumo de riesgo - relacionados casi siempre con el ocio- ha conducido a concebir que no puede hablarse de un rechazo generalizado del riesgo en nuestras sociedades de consumo, sino que habría que hacerlo de distintos estilos de riesgo; es decir, de distintas percepciones del riesgo y comportamientos hacia el mismo, no pudiéndose generalizar su aversión, según esta perspectiva. Sin embargo, una mínima mirada a las instituciones refleja el crecimiento de las destinadas a la disminución de los riesgos del consumidor: desde las leyes a los centros o institutos de protección del consumidor, pasando por

\footnotetext{
${ }^{6}$ Como muestran Ritzer y Liska (1997) para el montañismo, a pesar de establecerse discursivamente sobre la falta de racionalización, concentra comportamientos muy racionalizados aplicados al control.
} 
RIS

las asociaciones o las agencias de seguridad alimentaria ${ }^{7}$. Así, las organizaciones y asociaciones de consumidores han aumentado su peso institucional en función del argumento de la protección ${ }^{8}$. Mientras las relaciones laborales viven un proceso en el que las normas se dirigen a debilitar la protección de los trabajadores (contratos indefinidos, garantías frente al despido, subsidios de desempleo), el consumo se llena de instituciones y reglamentaciones protectoras.

\section{DISTINTOS NIVELES DE RELACIÓN ENTRE RIESGO Y CONSUMO}

La afección del consumidor a la seguridad invita a una doble lectura: la introducción del riesgo en el consumo es un riesgo para la sociedad de consumo; o se ha transformado de tal manera la producción social del consumidor, que éste aparece más inclinado a aceptar el riesgo. Esto conlleva admitir, con Marx, que el consumidor, como la mercancía, está socialmente producido. Pero antes conviene entrar sintéticamente en las distintas relaciones analíticas entre riesgo y consumo. Para ello, se establecen tres niveles de la relación entre ambos términos ${ }^{9}$ :

A) Nivel de riesgos metamercantiles: Condicionan el conjunto del consumo y son difícilmente reductibles a mercancía. Son los que están en la producción social de la mercancía: desequilibrios económicos, inestabilidad económica, paro, etc., existiendo otros relacionados, como el desarrollo de la higiene, las infraestructuras, la información, etc.

B) Nivel de los riesgos mercancia o mercancias seguridad: Campo creciente, intenta engullir riesgos que eran metamercantiles (salud, vejez, desempleo) en las sociedades del bienestar. Una de las transformaciones importantes es la progresiva expansión de estas mercancías, conduciendo a la seguridad como valor de cambio: se compra y se vende seguridad. En la medida en que este tipo de mercancías se proyecta de manera desigual sobre el conjunto de la sociedad, es aquí donde cabe hablar de estilos de riesgo, es decir, en la mayor o

\footnotetext{
${ }^{7}$ La agencia española se crea en septiembre de 2002, casi al mismo tiempo que otras europeas.

${ }^{8}$ Las asociaciones de consumidores se institucionalizan como un mediador capaz de presionar al Estado. Ahora bien, no lo hacen tanto desde la defensa de los derechos atribuidos a los consumidores, como desde la denuncia de la posición de poder de los productores y distribuidores. Son organizaciones que se mueven a partir de las denuncias del consumidor, pero que no mueven al consumidor, independientemente de su "problema organizativo de movilización" (Offe, 1990:227).

${ }^{9} \mathrm{Se}$ dejan a un lado los peligros, sobre los que no hay nada que decidir, salvo algunas precauciones. Entran aquí: catástrofes naturales, atentados terroristas, guerras, etc., sucesos que no suelen cubrir las compañias de seguros. Por lo tanto, de entenderse como riesgos, no entran por el agujero de la forma mercancía.
} 
menor inclinación o demanda de los grupos sociales a esta mercancía en función de su posición en la estructura social. Es una inclinación que, no obstante, ha de explicarse por el grado de extensión del nivel de los riesgos metamercantiles, pues en la medida en que éstos se encuentran cubiertos fuera del mercado, entrarán menos en el juego de la distinción en el consumo. El proceso parece ir en sentido contrario, ya que la relación entre seguridad y riesgo tiende a convertirse en un elemento estructurado de los estilos de consumo y, por extensión, de los estilos de vida. Es lo que permite hablar de estilos de riesgo, como integrados en estilos de consumo.

C) Nivel de riesgos especificos de las mercancías: Son riesgos derivados del uso de las mercancías, y admitidos en la medida en que se admitan como mercancías (valor de cambio de las mercancías). Aquí el margen de los riesgos es amplio, pues va desde que no funcione un artefacto que hemos comprado, a los accidentes de tráfico, que son relativamente aceptados en la medida en que la mercancía coche tiene importante valor signo y simbólico. La relación entre consumo y riesgo en este nivel, interior a la mercancía, se encuentra en la polaridad entre confianza y desconfianza, un extenso intervalo en el que intervienen distintos agentes, siendo la marca el que destaca ${ }^{10}$.

Las transformaciones en la relación entre riesgo y consumo pueden explicarse a partir de la estructuración de estos niveles de riesgo, teniendo en cuenta que A condiciona a B y B condiciona a $\mathrm{C}$, en el comportamiento de los sujetos. Son niveles jerárquicos. Es el nivel A el que principalmente se ha modificado. La producción social de la mercancía se realiza con un creciente riesgo (competitividad, precariedad en el empleo), sobre todo, en el ámbito de las relaciones de producción. Hay una producción social de riesgo en la producción de la mercancía; un aumento en la producción social del riesgo que cabe atribuirlo al propio modo de acumulación flexible (Boyer, 1986).

$\mathrm{Al}$ producirse en $\mathrm{A}$ tal proceso de reducción del ámbito de las seguridades, B aumenta incluso ganando terreno a A: se expulsan seguridades de A y se mercantiliza lo que antes era metamercantil. En la medida en que aspectos de la inseguridad contextual se quiebran, se abre el campo para la mercantilización de la seguridad. La seguridad aumenta así su presencia entre las mercancías y servicios de consumo, incorporando la dimensión riesgo en el propio consumo. Introducir como mercancía la seguridad en consumo, exige introducir también el riesgo, la señalización del riesgo, como explícita o implícitamente hace la publicidad (sólo hace falta observar la publicidad sobre seguros de pensiones).

\footnotetext{
${ }^{10}$ En un estudio realizado tras el 11 de septiembre de 2001 sobre la percepción de los viajes en avión, se pudo observar el poder, casi mágico, que tenían algunas marcas, frente a otras; un poder basado en la historia de la relación entre consumidores y la propia marca. La historia, también en el ámbito del consumo, surge como ese inconsciente colectivo.
} 


\section{RIS}

Si a ello se añaden las periódicas crisis experimentadas en algunos campos del consumo, como el alimentario; pero, también, en otros como, por ejemplo, el automovilístico (el caso Firestone) o el de las telecomunicaciones (el caso de la ubicación de antenas para telefonía móvil), nos situamos ante un consumo que es, a la vez, fuente de riesgo y solución de riesgos. Surge, así, el consumo como generador de riesgos que sólo el consumo, con la decisión del consumidor y el argumento de la soberanía del mismo, tiene en sus manos satisfacer. Tal vez sea en el discurso publicitario donde mayor evidencia tenga esta situación, pues el gran reto de la publicidad es ganarse la confianza de los consumidores. Algo que crecientemente se ve obligada a hacer sembrando dudas y desconfianza.

El modelo de partida de la relación entre riesgos y consumos, el que puede enmarcarse como característico de la sociedad de consumo, es el que requiere un amplio abanico de seguridades en $\mathrm{A}$, ofreciendo un gran margen de seguridades fuera del mercado. La sociedad de consumo emerge a partir del relativo control de ciertos riesgos (salud, vejez, enfermedades, etc.).

El propio sistema de consumo ha hecho que vaya en aumento el peso de B, incluso llegando a la sensación de que podía llegar a abarcar a $\mathrm{A}$ y que todas las seguridades pueden entrar en el mercado. Ahora bien, para ampliar el margen para la producción de la mercancía "confianza", hay que ampliar, a su vez, la producción social de riesgos (Zucker, 1986). Al mercantilizarse progresivamente, A pierde su fuerza y su legitimidad bajo B (el mercado aparece ofreciendo mejores niveles de seguridad) ${ }^{11}$ y $\mathrm{C}$ (el consumidor elige su nivel de riesgo).

La extensión del dominio de la mercancía "seguridad" (B) se realiza ganando terreno al espacio de las seguridades que eran puestas fuera del mercado (A), con las consecuencias sobre el propio consumo. Se empiezan a mostrar límites al propio consumo: ¿Es posible sostener este desequilibrio entre áreas de seguridad/riesgo en el consumo? Los riesgos empiezan a generar incertidumbre sobre el consumo. Las crisis periódicas no han impedido la sensación de que: "estamos entregados, casi sin protección a las amenazas industriales de la segunda naturaleza incluida en el sistema industrial. Los peligros se convierten en polizones del consumo normal" (Beck, 1998:13). Integrado el riesgo en el consumo normal, se ha establecido como una de sus mercancías principales.

\section{DE LA ELECCIÓN A LA DECISIÓN: EL DISCURSO DE LOS SUJETOS}

El análisis de la producción social del riesgo observa las elecciones como determinadas, lejos de una supuesta autonomía del consumidor. En los niveles presentados

\footnotetext{
"Véase el impulso del mercado de pensiones ante las amenazas sobre el sistema público de pensiones.
} 
anteriormente, sólo hay márgenes para la relativa elección autónoma del consumidor en los niveles $\mathrm{B}$ y $\mathrm{C}$.

Tanto Beck (1998), como Luhmann (1998) y Douglas (1998), o, entre nosotros, Beriain (2000:87), presentan el aumento de riesgo en nuestras sociedades como consecuencia perversa del proceso de modernización ${ }^{12}$. Beck (2002) nos habla de la conciencia de las diversas consecuencias no deseadas de la modernidad radicalizada, Luhmann (1998), de diferenciación y sociedad compleja, Douglas (1998), de distancia de la comunidad. Surge en los tres autores, el aumento del riesgo como la factura social que hay que pagar por la individualización, pero como si tal individualización fuese la fatal o afortunada consecuencia de un proceso autónomo, como si la individualización fuese también un producto social, lo que deja entre paréntesis, al menos, la asimilación de la misma a un logro, que lleva a los sujetos al universo de las elecciones y las decisiones.

Habría que preguntarse si los sujetos, en los comportamientos que viven desde la dimensión del riesgo, lo establecen desde un marco de decisiones o elecciones individuales, o desde algunas de las concreciones, tal vez modernas, de la fatalidad; además de cuestionarse si lo hacen desde la conciencia de una individualidad autónoma o heterónoma ${ }^{13}$.

Vaya por delante que las distintas aproximaciones empíricas realizadas dibujan unos sujetos lejos de sentirse arrojados, como individuos aislados, en marcos de elección. Principalmente, aparecen como individuos arrojados a la fatalidad, a estrechos marcos de elección y con la conciencia de que son decisiones con proyección irreversible sobre su identidad.

Estas afirmaciones derivan de distintas observaciones empíricas, que, modestamente, han atravesado la estructura social. Son estudios que han abordado objetos de investigación inscritos total o parcialmente en el campo del consumo, pero que, a la hora de presentar aquí sus resultados, desestimaremos aquéllos que, aun yendo en el mismo sentido, se insertan en estudios centrados en el ámbito productivo (Callejo, 1996).

\footnotetext{
${ }^{12}$ De manera coral en Giddens, Bauman, Luhmann y Beck (1996), o Lash, Szwerszinsky y Wynne (1996).

${ }^{13} \mathrm{Al}$ respecto, hay que señalar la ambigua relación que establece Beck (2002) entre elección y autonomía. Por un lado, parece ser el proceso de individualización el que lleva a los sujetos a una constante toma de decisiones y elecciones (o no elecciones); pero, por otro lado, subraya que se trata de una individualización "no autónoma", donde habría elecciones sin posibilidad de elegir, sin repertorio. Es lo que denominamos aqui decisiones. Así, la individualización de Beck (2002), como él mismo señala, se convierte precisamente en su contrario. Algo que, por otro lado, parece casar mal con su idea de biografias autoproducidas. Si no hay elecciones, ¿cómo puede autoproducirse la biografia, que se supone realizada en condiciones de libertad de elección?
} 
RIS

REVISTA INTERNACIONAL DE SOCIOLOCIIA

№ 40, ENERO-ABRIL, 2005

JAVIER CALLEJO GALLEGO

1) El primer estudio tiene lugar en el mes de enero siguiente a los atentados de Nueva York del 11 de septiembre de 2001. Su objetivo era sondear la disponibilidad a volar en avión tras tales acontecimientos. Es decir, entraba directamente en el análisis de la percepción de riesgo a volar ${ }^{14}$, cuando el "consumo de avión" es una práctica extendida en la mayor parte de las capas sociales de las sociedades avanzadas.

La investigación se llevó a cabo entre individuos de clase social media y media-alta. Pues bien, en este sector social, que cabría esperar se inclinase por actitudes guiadas por la racionalización, se encuentran notables rasgos de fatalismo, es decir, de asunción de situaciones de riesgo descritas sin capacidad de acción ni de elección ${ }^{15}$. Se asume el riesgo pero porque no se puede salir de él, pues es parte de sus condiciones de producción (de su trabajo), especialmente entre el grupo de ejecutivos de Santiago de Compostela o Barcelona (ver nota 14). Se sigue volando. La elección se traslada a la marca, configurándose unas como más seguras que otras y, al contrario, unas como menos seguras que otras: "Hay compañías hoy en día, Aerolíneas Argentinas o America Airlines, British ahora un poco más, pero cuando estaban en guerra yo las esquivaba porque estaban cooperando mucho" (RG. P. Mallorca) (ver nota 14). Conociendo el riesgo y no pudiendo salir de él, la disonancia se aplaca con la racionalización. Así, como dicen en el grupo de Barcelona: "Tampoco hay que tener tanta psicosis porque realmente, por muy grave que sea, en comparación con todo lo que hay, no han sido todos los muertos que tenemos cada fin de semana en las carreteras y todo el mundo cogemos el coche cada día" (RG. Barcelona) (ver nota 14). Al no poder salir de los consumos de riesgo, se sale de sus alarmas.

2) El segundo estudio ${ }^{16}$ se aproxima más a las clases populares. Su objetivo, dentro de un estudio más amplio, era construir un mapa de concepciones de la

\footnotetext{
${ }^{14}$ El diseño metodológico consta de cuatro reuniones de grupo; 1) RG.1: Varones de clase media-alta, que habitualmente viajan al extranjero para sus vacaciones (al menos lo habían hecho los dos últimos años), la mitad de ellos con hijos, entre 40 y 55 años, realizado en Palma de Mallorca; 2) RG.2 Ejecutivos y profesionales de grandes empresas que viajan frecuentemente al extranjero (una vez al mes, como mínimo), de clase social media-alta y edad entre los 35 y 50 años, realizado en Barcelona; 3) RG.3 Empleados y funcionarios en relación con el público, de clase media, entre los 25 y los 35 años, realizado en Las Palmas de Gran Canaria; 4) RG. 4 Empleados y trabajadores autónomos, que realizan frecuentes viajes en avión por motivos laborales, clase social media-media, entre los 35 y los 55 años, realizado en Santiago de Compostela.

${ }^{15}$ Cuando el consumo es opción, ante la presencia de riesgo entonces sí aparecen las elecciones: "Yo sí que tengo miedo. Tenía previsto un viaje a Argentina y pensar en tantas horas de vuelo (...) No tenía nada concreto, tenía más o menos las fechas, pero pensé: 'bueno, ya lo haré'. Nunca me gustó mucho volar" (RG. P. Mallorca).

${ }^{16}$ Gómez, Callejo y Aboitiz, "Factores sociodemográficos y sociales que determinan la supervivencia diferencial de la población española en el umbral del siglo XXI" (PB96-0154), financiado por la Subdirección General de Formación y Promoción del Conocimiento del MEC.
} 
salud ${ }^{17}$. Destaca en este estudio la vinculación del estado de salud con las condiciones laborales, sobre todo, entre los prejubilados y los empleados varones; un ámbito en el que los individuos se perciben sin capacidad de elección, incluyendo la rescisión de contrato, como ocurrió entre los prejubilados. En el conjunto de los grupos, la salud aparece, con mayor o menor intensidad, identificada con el dolọ y la enfermedad, sin que haya lógicas de previsión. Como se dice, "cuando aparece, aparece". Lo que podría considerarse la salud perfecta es algo que se disfruta y que, por lo tanto, no se tiene en cuenta para poder disfrutarla con mayor intensidad. Se encuentra presente, hasta que se agota, por una especie de vital fatalidad. Es algo que se acaba con el tiempo, no percibiéndose que el sujeto tenga algo que ver con ello.

Un análisis detenido de los discursos producidos muestra la subyacente presencia de una estructura que, en principio, pudiera parecer extraña. Se trata de la estructura organizada a partir de la polaridad dentro-fuera. Una polaridad que nos remite, en un inicio, al control, a las percibidas posibilidades de control sobre su salud -y sobre los otros miembros de su familia-por parte de los individuos. Dentro es un espacio donde los individuos pueden ejercer cierto control sobre su comportamiento y su salud; es un espacio de salud o, al menos, un espacio relativamente protegido de las agresiones externas a la salud. La salud está en casa, se busca en casa y, sobre todo, en el consumo. El afuera aparece casi siempre como una fuente nociva para la salud; desde la contaminación atmosférica, a partir de la polución fabril o automovilística, a la tertulia en el bar, donde se fuma, se bebe y hay ruido; es el espacio simbólico en el que se sitúa lo ajeno al control de los sujetos.

En el adentro los individuos pueden tomar medidas, desde el descanso a la dieta alimenticia. En el imaginario, el adentro es una especie de burbuja. En el afuera (el exterior), se establece la sensación de fatalidad, de que se depende de fenómenos ajenos a la propia voluntad. Es más un adaptarse a las circunstancias, que intentar prevenirlas; en el imaginario, el afuera cunde el peligro para la salud. En el exterior, no hay individuos ni elección.

La relación entre el adentro y el afuera no es estática. Es más, uno de los procesos señalados por los consultados es lo que podría denominarse creciente

\footnotetext{
${ }^{17} \mathrm{El}$ diseño metodológico comprende seis reuniones de grupo entre clases populares, con la excepción de la reunión cuarta; RG.1) mujeres entre 18 y 20 años, con empleo o experiencia laboral, realizado en Madrid; RG.2) varones entre 21 y 25 años, sin estudios universitarios terminados, la mitad en actividad laboral habitual, fumadores, realizado en Madrid; RG.3) varones entre 55 y 67 años, prejubilados del sector industrial, realizado en Valladolid; RG.4) mujeres entre 35 y 45 años, entre clases medias profesionales, la mitad con hijos, realizado en Valencia; RG. 5) varones entre 48 y 55 años, empleados, comerciales, etc., especialmente en el sector privado, al menos la mitad fumadores, realizado en Madrid; RG. 6) mujeres entre 45 y 55 años, sin actividad laboral extrafamiliar, realizado en Tordesillas.
} 
RIS

REVISTA INTERNACIONAL DE SOCIOLOCIA

$\mathrm{N}^{\circ} 40$, ENERO-ABRIL, 2005

dominio del afuera sobre el adentro, siempre en relación con la salud, algo que principalmente viene de la mano de la alimentación. Como se dice en los grupos, ya no se sabe qué se compra y se come, lo que genera un sentimiento de desprotección y fatalismo, de tener la salud en manos de "otros". De este modo, mensajes sobre prevención o, en general, cualquier control de la salud, pueden encontrar un campo abonado en su contra. Es decir, se sabe de la importancia del cuidado de la salud, pero también se siente un margen de actuación muy limitado y, lo que tal vez sea peor, dependiente. No es tanto que no se sepa qué hacer, sino que no se sabe qué hacen los demás. Ese afuera se instala hasta el adentro, espacial y simbólico, y hasta el espacio interno, corporal, a través de la alimentación. Es un fatalismo que anula la deslegitimación de comportamientos de riesgo para la salud relacionados con la ingesta-fumar, beber alcohol, drogas-y deja un tanto en el vacío los mensajes institucionales sobre la prevención. Hay que señalar que tales percepciones de los grupos se recogieron pocos meses antes de que se produjera la denominada "crisis de las vacas locas" en el conjunto de Europa Occidental.

No estando en una posición social estable —ni laboral, ni económicamente, ni en sus propias relaciones personales-, y con un horizonte de movilidad social, imaginariamente amplio en algunos casos, su estilo de vida se concreta en la casi disponibilidad absoluta a las demandas externas. Una disponibilidad soportada por el cuerpo: "Hasta que el cuerpo aguante". Algo especialmente acentuado entre los jóvenes ${ }^{18}$. No es que no haya conciencia de la salud, es que sus circunstancias sociales, la alta disponibilidad a las demandas externas, les impide la práctica de tal conciencia. Tenerla supondría un freno, una menor disponibilidad. Es la presión la que marca las decisiones, sin que haya margen de decisión.

Intentando destacar el eje central y común de las concepciones de la salud observadas en las reuniones de grupo realizadas, tal vez puedan sintetizarse en la doble articulación desubjetivadora que une condiciones concretas y fatalidad. Los grupos achacan la situación de su salud en relación a las condiciones vitales que les ha tocado vivir, como si el margen de las alternativas de la acción fuese mínimo. Frente a unas condiciones que generan estrés o que anulan la identidad del individuo, unas condiciones que exigen aprovechar la oportunidad laboral o que intensifican los ritmos y, sobre todo, la obtención de resultados, los sujetos se ven incapaces de dar preferencia a la salud y a la información que tienen sobre los riesgos que pueden conllevar ciertos comportamientos.

No es carencia de información sobre los aspectos que pueden poner en peligro la salud lo que se ha percibido. Es más, en unos grupos por interés sobre la temática (como ocurre con los grupos más adultos), en otros, por su mayor información sobre los más diversos aspectos de la realidad social (como ocurre

\footnotetext{
18 "Sí. Más o menos. Sí, que tu cuerpo sea capaz de aguantar tu ritmo de vida diario. Sea cual sea el que hagas, dependiendo de tus circunstancias..." (RG. Chicas, Madrid).
} 
con los grupos de jóvenes), la información sobre riesgos relativos a la salud circula con relativa facilidad, $\mathrm{y}$ recoge rápidamente ecos constantes. El problema es el margen de acción que les queda a los individuos para, una vez conocidos los riesgos, llevar a cabo conductas de no riesgo.

El principal ámbito de racionalización y justificación, de la asunción de los riesgos, hay que buscarlo en la referencia a un contexto productivo-laboral en veloz cambio, tan repleto de oportunidades, como de amenazas de desplazamiento definitivo, donde la necesidad de adaptarse se convierte en requisito de supervivencia. Hay que hacer notar que el contexto productivo, como fuente de riesgo para la salud, no viene dado por condiciones físicas adversas, por las descripciones que realizan los jubilados de Valladolid (ver nota 17), establecidas sobre posiciones corporales nocivas. Ya no se trata de la cadena productiva y del encadenamiento, no buscado, pero referido como inevitable. No se trata de una salud asalariada. Ahora nos encontramos con un contexto productivo dominado por la búsqueda autónoma de aprovechamiento de la oportunidad.

Parece imposible la planificación en un contexto productivo de continuo cambio, donde no hay vínculo contractual, ni, por lo tanto, posición productiva medianamente estable, ni, lo que tal vez sea más importante, vínculo sólido con los derechos derivados de la condición asalariada ${ }^{19}$. Es más, los concebidos como pequeños riesgos ("pequeños vicios") se constituyen en compensación de los riesgos mayores, (como la ansiedad en el caso del tabaco), o, al menos, mínimos peligros que, además de generar satisfacción, poco parecen añadir a los peligros mayores, (como ocurre con el tabaco en comparación con la contaminación) ${ }^{20}$. La racionalización sobre la inevitabilidad de algunos aspectos relacionados con la (mala) salud, lleva a asumir como inevitables otros comportamientos sobre los que tal vez se podría llevar a cabo alguna decisión, como los relacionados con el consumo.

La estructuración que hemos dibujado, polarizada entre lo evitable y lo inevitable, deriva directamente de los discursos de nuestros grupos. Hablar de la salud en términos de inevitabilidad, casi de fatalidad, supone, a la vez, un cambio y una regresión. Una regresión en cuanto significa volver a la propia fatalidad, a que los individuos dejen sus comportamientos en manos de la suerte. Un cambio, en cuanto adquiere nuevas tonalidades. Ya no es una fatalidad regida por designios divinos, sino que se encuentra producida por el propio hombre, siendo el resultado de la

\footnotetext{
19 “Tú, por ejemplo, estás en un contrato de empleado, seis meses, vale; llega al final y no sabes si te van a renovar o no" (RG. Chicos, Madrid)

20 “...Te sienta bien porque esa ansiedad te la mata, pero indudablemente que no es salud, ni uno, ni tres ni ninguno.Tú vas por la calle y te comes el humo de un coche, o el de la furgoneta y te contamina más, por supuesto" (RG. Mujeres, Valencia). Situaciones que pueden denominarse de riesgo saturado, donde un comportamiento habitualmente de riesgo no añade más riesgo a la situación.
} 


\section{R I S}

acción humana en favor del progreso. Es una especie de fatalidad del progreso y en el progreso.

- "Hay una cosa cierta. Estamos hablando de cosas que son inevitables. De la salud. Yo la entiendo de dos maneras. $O$ hay dos modalidades de salud: la que es inevitable, o la que te imponen, alimentos, contaminación. Eso no lo podemos evitar, por más que hablemos. Si la fruta no sabe a nada, si los huevos, no son huevos, si la carne, no es carne, si los coches contaminan...

- Sí, sí

- ...o sea que, son cosas inevitables. Pero, hay otro factor que es el que se puede evitar, que es al que se refería, me parece este señor. Entonces, lo inevitable no podemos evitarlo, porque está ahí. $\mathrm{O}$ sea, no podemos aislarnos en una isla, tenemos que vivir con lo que tenemos..." (RG. Varones 48-55).

Es la acción colectiva del hombre y sus resultados, el progreso, la que reduce el campo de la acción individual con relación a la salud. De esta manera, surge con cierta contundencia lógica el que la gestión de la propia salud se deje en manos de lo concebido como elemento puntero del desarrollo del propio progreso: la ciencia en general y la medicina en particular. En un contexto de desconfianza,hay que confiar en algo.

Si aparece fuera de la lógica el sacrificio de los considerados pequeños placeres, aunque se tenga información sobre su carácter nocivo, menos cabe tener reparos en poner en juego la propia salud cuando de lo que se trata es de aprovechar las oportunidades en el ámbito productivo o, como ocurre en las posiciones relativamente estables y subordinadas en la estructura productiva, de lo que se trata es de adaptarse a las presiones derivadas de la racionalización productiva, donde desaparece todo margen de elección:

“- ...Yo llevo veinticinco años y ha cambiado dos veces de los bancos, se hace el mismo producto. No te exigían tanto. Antes, eran americanos y ahora son franceses. Los americanos te exigían su producción, pero no te exigían tanto como te exigen ahora los franceses. Los franceses ahora mismo van a estrujarte. Y cada día más y más. Se están dando casos de ataques al corazón y de muertes, así. Y eso, está creando...

- Y enfermedades mentales...

- ...ataques al corazón. Y ya hay varias personas que han muerto, porque es que están machacando cada día más y exigiéndote y venga. Antes, podías decir: Oye, que es que tal. Pero, es que ahora, no puedes ni abrir la boca..." (RG. Varones 48-55).

Si la racionalización productiva de hace medio siglo venía dada por la cadena de montaje y la conversión de los cuerpos de los trabajadores en prolongaciones de la maquinaria industrial, nos encontramos ahora con la competencia generalizada y, sobre todo, con el requerimiento de tener que demostrar continuamente la propia 
competencia. La disponibilidad productiva constante, representada sobre todo por la figura del profesional autónomo, es contraria a las demandas para la protección de la salud. Tal disponibilidad se encuentra especialmente acentuada en el caso de las mujeres profesionales que, además, se encuentran con la obligación de ser responsables de la gestión doméstica:

-”... En casa siempre tienes la obligación de que o te tienes que levantar, preparar no sé qué... Ojalá tuviéramos ese tiempo. Sobre todo las que trabajamos a mediodía... Aunque tengas dos horas, da igual.

- Es que las tienes que emplear en otras cosas.

- Llegas y te pones a ordenar antes no sé qué que a comer.

- Sí, sí

- Bueno, yo creo que eso también depende del turno de comidas, si comes sola, si comes con tus hijos o con tu marido, si coincides... Porque yo cuando como sola es evidente que ni me siento, y cualquier cosa.

- Que vas comiendo de pie.

- Exacto.

- Vas picando.

- Voy por casa preparándome el bolso, el monedero y tal, y de paso voy picando. Pero normalmente como coincido en el horario con mi marido... Tengo dos turnos de comida, primero comen mis dos hijos, que tienen un horario, y entonces yo lo espero a él y comemos los dos. Entonces, como él es muy tranquilo y muy relajado, ya me he acostumbrado a que..."(RG.Mujeres, Valencia).

Vemos cómo la alimentación, que es uno de los principales instrumentos de gestión de salud en lo cotidiano, se deja a un lado. No hay tiempo. La fatalidad en el riesgo, aunque parezca contradictorio, genera una espiral acelerada que no parece poder pararse, como muestra especialmente el discurso de los jóvenes varones. Un riesgo lleva a otro riesgo, a un estilo de vida en el riesgo. La disponibilidad a la flexibilidad y la oportunidad exige estar donde surgen las oportunidades. $\mathrm{La}$ cultura de la oportunidad es, en buena medida, un resultado del modo de acumulación flexible. Los sujetos más jóvenes entre los consultados no aparecen estor dispuestos a abandonar la lógica de la oportunidad. Su margen de maniobra al respecto no es amplio. Es su forma de adaptación a las condiciones actuales.

En el ámbito del consumo, la relación entre alimentación y salud se encuentra bajo sospecha. Aun así, sigue siendo el ámbito preferente en el que los individuos, especialmente las mujeres ${ }^{21}$, perciben que pueden construir salud. Algo importante si se tiene en cuenta la reducción de tales espacios para la construcción de la salud, incluso la pérdida de tal posibilidad de construcción. El riesgo dentro del consumo es alto, pero aún es mayor fuera de él y menor la posibilidad de elección. Mejor el control alimenticio, susceptible de convertirse en un control sobre la salud, que la percepción de un descontrol ajeno sobre la alimentación, 


\section{RIS}

que repercute en un descontrol propio sobre otros aspectos de la salud. He aquí el lugar clave de la alimentación. Las sospechas sobre la misma abren la veda a todo tipo de conducta insana, pues, entonces, no hay refugio:

-"...Una fruta que sabe, que en cuanto la dejas dos días te sabe a insecticida. Que dices: Bueno, aquí si yo no echo insecticida y esto, no hay quien lo coma. Y lo tienes que tirar. Entonces, no estás comiendo. Estás manteniendo una orientación. Pero, no estás comiendo realmente. No te estás alimentando. Y quien dice eso, dice temas más serios que eso. Es el tema que ha apuntado el compañero de la contaminación atmosférica del aire. Porque eso, ya sí que no vale...

- Y la alimentación

- ..Y la alimentación. Pero, es que una cosa está con la otra. Porque el aire incide en la producción, y que vayas al campo y te encuentres con árboles en plena época. Y que digas: ¡Pero, qué estoy viendo!. Si estoy viendo que no tiene fuerza, el árbol en ésta época. Estoy viendo que están las hojas medio podridas...

- ...Sí, sí. Está todo. Pero bueno, estamos metidos en un mundo de la alimentación. Entonces, el tristemente caso del aceite de colza, en fin. Estamos comiendo cosas que, no sabemos lo que estamos comiendo. Que nos están envenenando...

- Nos estamos contaminando" (RG. Varones 48-55).

La contaminación alimenticia se convierte en metáfora de la imposibilidad de acción en clave de salud, en afirmación de que todo está contaminado, de que no quedan márgenes para la actuación con respecto a la prevención y cuidado de la salud, excepto la curación o el mantenimiento artificial de la vida. Así, nos encontramos que el consumo - especialmente el consumo alimentario- se convierte en otra fuente de riesgos más, junto a las otras; pero, a la vez, también aparece como uno de los pocos campos en los que todavía cabe algún control y, por lo tanto, una acción relativamente autónoma. Ahora bien, no deja de ser una acción bajo una fatalidad contextual. Por esto, cabe hablar de decisiones y no de elecciones.

3) El tercer estudio empírico que aquí se presenta trata directamente del ámbito del consumo. Aborda el consumo alimentario de los "nuevos hogares", hogares que por diversas circunstancias (matrimonios, emancipación de jóvenes, enviudamiento, etc.) han de plantearse o replantearse sus hábitos

\footnotetext{
${ }^{21}$ La alimentación se considera la forma de construir salud por parte de las mujeres: "Bueno, yo soy Marta. Soy una pequeñita empresaria, muy pequeñita. Soy fumadora empedernida, como tú; tomo mucho el sol; no hago ni pizca de ejercicio, aunque no paro, aunque no paro. Aunque el ejercicio físico que hago supongo que me hará polvo. Y bueno, me dedico a cuidar a mis hijos también, eso también es importante. Yo por lo menos, a ellos les voy dando otros hábitos, otro tipo de alimentación a lo mejor de la que como yo, que como deprisa y corriendo para seguir la marcha. En fin, yo la salud desde luego la tengo muy en mente para los demás, para mí no.

- Sí, como yo" (RG. Mujeres, Valencia).
} 
alimentarios ${ }^{22}$. En principio, no parece un marco para dudar de la existencia de elecciones hechas por los individuos. Pues bien, la dominante distancia entre el ideal de los hábitos alimentarios y lo que se hace, especialmente en los hogares jóvenes, muestra un escaso margen de elección. Un margen limitado especialmente por la falta de saber hacer (socialización en la gestión y preparación de los alimentos) y las condiciones de trabajo.

Se eligen relativamente los alimentos, pues estamos ante sectores de la clase media con cierta capacidad adquisitiva, pero no se eligen los estilos de consumo. Incluso se realizan algunos comportamientos de consumo desde la propia conciencia de riesgo, de ciertas sospechas que fundamentalmente aparecen cuando se habla de la comida de los niños. Pero, aun así, son más potentes los condicionantes que impiden un consumo más sano, que es lo que se quisiera elegir.

"Pero no me siento bien en lo que le estoy dando, o sea ¿sabes? Me siento mejor cuando le doy una croqueta que sé que la he hecho yo, antes de comprar una cosa preparada, lo que pasa que claro, para hacer lo que le estoy dando de comida necesito tiempo, y ese factor tiempo pues ahora mismo no lo tengo" (Administrativa, 30 años, dos hijos, Valladolid).

Condicionantes como la falta de tiempo e, incluso, la propia comodidad, reducen las alternativas de acción de los individuos. ¿Se deciden riesgos? No, parece que, a lo sumo, se aceptan riesgos; pero que, en lugar de generar culpabilidad en los sujetos, absuelve a éstos de la misma, ya que no la viven como una decisión propia, sino como decisiones fruto de sus condiciones de vida, vinculadas con las condiciones de producción (horarios, cansancio, etc.).

Cuando no hay tiempo, se practica la denegación del riesgo. La práctica más consonante es negar el riesgo, aun cuando, contradictoriamente, se reconozca desde la situación reflexiva de la entrevista y, entonces, se reduzca su relevancia. Cuando hay tiempo, como se reconoce en una entrevista a una maestra jubilada con conocimientos y hábitos culinarios, la elección también aparece como una ficción, pues los productos sólo lo son de nombre, por el significante: un tomate sólo lo es por el nombre ${ }^{23}$.

Cuando se está en condiciones de riesgo - en función de las condiciones de vida y, sobre todo, de las condiciones de estar en la producción - aparece el riesgo en el consumo. A la vez, la sospecha y la necesidad de confianza. Es aquí, en la

\footnotetext{
${ }^{22}$ Cecilia Díaz (investigadora principal): "Cambio en el consumo alimentario en España", financiado por el Plan Regional de Investigación del Principado de Asturias (PB-EJS01-15). Los discursos se produjeron en seis grupos de discusión entre mujeres (Barcelona, Oviedo y Valladolid) y una cincuentena de entrevistas repartidas entre las tres áreas metropolitanas referidas.

23 “Ahora podemos elegir, podemos escoger, tenemos más variedad, nos engañamos si pensamos que ahora... antes era todo más real, un tomate era un tomate, y había madurado allí y te lo comías, y ahora un tomate, cuando te llega a la mesa, igual hace dos meses que está viajando por este mundo de dios" (Maestra retirada, 53 años, hijos fuera de casa, Barcelona).
} 
RIS

confianza en las marcas o las tiendas donde se establece entonces la decisión, pero una especie de decisión fatal, de encomendación a tal decisión:

"Pues yo creo que estamos comiendo mucha porquería, mucho producto adulterado, mucha hormona en, en todo, mucha fruta que no sabes si es un melocotón o un, un.. qué. O sea, que al final... Bueno, por ejemplo, tú la carne la puedes ver con buena pinta, pero en realidad no sabes todo lo que se ha tomado la vaca, como lo que ha salido ahora de las vacas locas. Y nosotros no... no, no vas a ir analizando las piezas. O sea, tú te tienes que fiar de la garantía que te den ellos. Si ellos no te dan esa garantía y por sacar más dinero o por lo que sea lo adulteran, pues, pues somos unos tristes inocentes" (Recién casada, 25 años, Oviedo).

Se trata de elecciones ciegas. Tal vez es a lo que se refiere Beck (1998) cuando habla de individuo "no autónomo", de elecciones sin autonomía. Realmente no hay elección, pues, para haberla, tienen que existir condiciones para realizarla autónomamente. Por lo tanto, nos encontramos que la escasa presencia de percepción de riesgo en el consumo se encuentra producida por las condiciones de vida de los sujetos, por las condiciones en que se produce el consumo.

Reducción del espacio de seguridad A, del espacio protegido generalizado tanto en la producción como en el consumo. Quienes pueden, por su posición en la estructura social y en la estructura de producción, compensarán la reducción con una mejor situación en $\mathrm{B}$ (comprando seguros) o en C (mercancías más seguras). Quienes no, verán cómo estas dos últimas áreas se agrandan y, como en parte ocurrió con las crisis del aceite de colza y de las vacas locas en España, vivirán culpablemente sus malas y pobres elecciones.

Las mercancías directamente relacionadas con el riesgo (nivel B), a través de la oferta de seguridad, han visto aumentar su relevancia ${ }^{24}$. Ello parece reforzar la constitución del consumo como un campo de seguridad, de refugio. Ahora bien, tal crecimiento se ha realizado a partir de la eliminación de buena parte de las bases de seguridad en las que se ha estado apoyando la sociedad de consumo durante la segunda mitad del siglo pasado. Es como si el consumo que tiene por base la búsqueda de seguridad creciese, como una especie de alien, en el cuerpo de una sociedad de consumo cada vez más llena de incertidumbres vitales, alimentándose de ese propio cuerpo. Las mercancías de seguridad crecen a costa de las condiciones en las que se producen las mismas.

Dentro del consumo, en la lógica de la mercancía, al situarse la seguridad como elemento de distinción, se denuncia acusadoramente la falta de seguridad de los productos de la competencia. La lógica de la distinción en el consumo, su ámbito principal, se fundamenta más en la señalización de la carencia de los otros

\footnotetext{
${ }^{24}$ Tanto en su peso relativo en los resultados de la Encuesta Continua de Presupuestos Familiares, como en los discursos sociales. En los primeros, el gasto en seguros de las familias ha pasado de un $2,69 \%$ en 1998, sobre el total del gasto anual, a un 3,03\% en 2002.
} 
(Bourdieu, 1998) que en la propia posesión. La racionalización de la carencia, que en el modelo de la soberanía del consumidor adquiere la forma de equilibrio entre precio y asunción de riesgo, toma las características de una necesidad hecha virtud: se consume con asunción de riesgos porque no hay otro remedio, aunque se vista de decisión racional, como ocurría con los viajeros de avión visto. Anteriormente: mientras se dice que el avión es el único medio en las distancias largas (se cita París en los discursos producidos), se asume que hay que viajar.

\section{CONCLUSIONES}

Desde la perspectiva de los sujetos, la relación entre riesgo y consumo sigue siendo relativamente débil, incluso en el consumo más sensible, como es el alimentario. Desde tal percepción, apenas podría habląrse de cambio de modo de consumo en la sociedad de riesgo, pues sigue siendo un ámbito de relativa seguridad. Ahora bien, ha cambiado la estructuración de los niveles de relación entre consumo y riesgo. En el denominado modo de acumulación fordista, la seguridad generaba consumo, mientras que en el modo de acumulación flexible es el consumo el que genera seguridad. Cuando surge el discurso del riesgo en el consumo, lo hace vinculado a las condiciones en las que viven los sujetos y en las que se producen las mercancías. La inseguridad y la incertidumbre se encuentran menos en el interior del consumo, que sigue creciendo como ámbito de seguridad y refugio, que en las condiciones de producción para el consumo.

Como se ha visto, en la lógica de la mercancía difícilmente entra el riesgo, aun cuando su presencia sea creciente en clave de seguridad. No obstante, cuando lo hace, como es en el caso de las crisis alimentarias, pone en evidencia un modelo de sociedad con un rostro que no se desea, señalando la necesidad de alternativas (Millán, 2002:283). Sin embargo, rápidamente parecen olvidarse estos acontecimientos críticos, recuperándose la concepción del consumo como un ámbito de seguridad, en un entorno de riesgo. Tales huellas de crisis y reclamación de alternativas, señalan la necesidad de pensar en otros modelos de consumo y, en definitiva, en otros modelos de sociedad. Pero es un paso que los sujetos consumidores que han formado parte de las observaciones empíricas no llegan a dar. Se encuentra el malestar, pero no el paso a cuestionar la sociedad de consumo, cuando es, a pesar del creciente riesgo que en ella se da, un oasis de seguridad en un contexto de incertidumbre. Domina la representación del consumo como un ámbito de seguridad, situándose la inseguridad en la producción.

El trabajo ha presentado tres distintos niveles analíticos en la relación entre riesgo y consumo. Pues, a la luz de los resultados empíricos, no son las mercancías, ni su uso, lo que genera riesgo, sino sus formas de producción, que se extienden a las mercancías producidas y a la producción de consumidores. Es el proceso 
de debilitamiento del nivel metamercantil en la relación entre riesgo y consumo, de las seguridades que se excluyen del mercado, lo que tienen en cuenta los consumidores. El riesgo no está en las consecuencias de las mercancías, sino en su forma de producir consumidores.

Por un lado, nos encontramos con que la expansión del riesgo en el ámbito de la mercancía, que es el del consumo, parece acelerar el consumo y el propio desarrollo capitalista. Como señala Beck: "la expansión de los riesgos no rompe en absoluto con la lógica del desarrollo capitalista, sino que más bien la eleva a un nuevo nivel. Los riesgos de la modernización son un big business. Son las necesidades insaciables que buscan los economistas. Se puede calmar el hambre y satisfacer las necesidades, pero los riesgos de la civilización son un barril sin fondo, insaciable, infinito, autoinstaurable" (Beck, 1998:29). Por ello, en las sociedades tardomodernas, la confianza se ha convertido en una mercancía (Silverstone, 2004:189). Lejos de acabar con el consumo, la expansión del riesgo lo acelera. La pregunta es: ¿puede sobrevivir como tal sociedad de consumo, una sociedad en la que el nivel de riesgo en la producción que la conforma es más elevado? ¿Puede la sociedad de consumo sobrevivir alimentándose sólo de seguridad en el consumo y, siguiendo nuestro esquema de relación entre riesgo y consumo, reduciendo la seguridad en las áreas de A (metamercantil) y $\mathrm{C}$ (seguridad de las mercancías)?

Sin las seguras bases del anterior modo de consumo, tal vez haya que empezar a trabajar desde el supuesto de otro modo de consumo, caracterizado por una mayor racionalización y, a la vez, una implicación reflexiva en la relación con las mercancías. La aversión al riesgo lleva a tener en el consumo un refugio; pero, a la vez, un refugio del riesgo y en el riesgo. Es un consumo en el riesgo y, como todo riesgo, es racionalizado. Puede hablarse hoy de un consumo reflexivo, que distancia al consumidor del objeto en una reflexión sobre sus consecuencias, frente al.consumo mágico de Baudrillard (1970) de la primera explosión de la sociedad de consumo; distancia y, a la vez, en cuanto refugio identitario en momentos en que la producción no genera identidades (Bauman, 1999), proximidad al consumo. Así, la principal diferencia entre el modo anterior de consumir y el que hoy se asoma es la misma que hay entre elecciones y decisiones, según se ha establecido aquí.

Donde la diferencia destaca es en lo que los individuos obtienen comparativamente en el trabajo y en el consumo. En un sistema productivo como el actual, en el que cada vez es más importante diferenciarse y distinguirse para sobrevivir, esto sólo se consigue con el consumo. Por ello, también el consumo se convierte en un ámbito de decisión vital. Del mundo mágico de la elección en un paraíso de la abundancia, se ha pasado a un mundo amenazante en el que hay que decidir.

El modo de consumo en un contexto de riesgo aparece con características más racionales, en el sentido que da Ritzer (1999), siguiendo a Weber. Como se ha visto en las aproximaciones empíricas, el consumo es un campo de más confianza que otros; pero, aún así, ya no es de fiar. La relación con las mercancías es menos impulsiva. 
Como no es de fiar, se demanda mayor mediación reguladora sobre el consumo. Mientras en la producción domina el fatalismo (hay lo que hay), incluso en su relación con la salud, en el consumo todavía aparecen en los discursos las decisiones: de los expertos en continua disputa (Giddens, 1997:112); del Estado para controlar (Bilbao, 1999:328); de los productores y distribuidores, y de los consumidores (Offe, 1990). Estamos en una sociedad de consumidores, a pesar de que el consumo no es lo que era; un cambio quizá demasiado leve para algunos como para reclamar la atención, pero que significa precisamente eso. El paso de la sociedad de consumo, como referencia de integración, a la sociedad de consumidores. Tal vez el paso por la literatura puede ser útil en la visualización del cambio. Se propone reflejarlo en el paso del trato diabólico de Fausto al de Dorian Gray.

En el modelo de Goethe, el consumo es la abundancia, es la posibilidad de elegirlo todo. Una posibilidad que se alcanza tras haber pasado por el trabajo y el esfuerzo de generaciones. De alguna manera, es el paso de la producción (profesión) al consumo y siempre - desde el principio (prólogo) al final de la obra-bajo la atenta mirada protectora de los cielos. Lo que acontece al doctor Fausto es un juego, una apuesta en el cielo y, como tal producto celestial, será protegido hasta el final. Basta una simple asimilación metafórica del cielo con el Estado de bienestar para entender la ubicación de este modelo literario en el inicio de la sociedad de consumo ${ }^{25}$.

Bajo el modelo de Oscar Wilde, algo envejece cuando se consume, como en el retrato de Dorian Gray. El consumo deja huellas -en la salud, en nuestras relaciones sociales, en nuestras relaciones laborales - aun cuando no aparezca en el instante inicial. En el modo actual de consumo, el pecado tiene una segura penitencia venidera que acaba en una muerte ensangrentada. Ahora, sin salvación final; con el único consuelo del recuerdo, tal vez fijado en un retrato de cuando éramos jóvenes y elegantes consumidores, de cuando decidimos asumir el relativamente apacible riesgo del consumo.

\section{REFERENCIAS BIBLIOGRÁFICAS}

AGLIETTA, M. (1979), Regulación y crisis del capitalismo, Madrid, Siglo XXI.

ALONSO, L. E. (2000), Trabajo y posmodernidad: el empleo débil, Madrid, Fundamentos.

BAUDRILlARD, J. (1970), La société de consommation, París, Denoel.

BAUMAN, Z. (1999), Trabajo, consumismo y nuevos pobres, Barcelona, Gedisa.

\footnotetext{
${ }^{25}$ Los profesores Lucas y Ortí vienen trabajando sobre el carácter fáustico del consumo. Antes, Baudrillard (1970).
} 
RIS

REVISTA INTERNACIONAL DE SOCIOLOGIA

No 40, ENERO-ABRIL, 2005

BECK, U. (1998), La sociedad del riesgo, Barcelona, Paidós.

(2002), La sociedad del riesgo global, Madrid, Siglo XXI.

BERIAIN, J. (2000), La lucha de los dioses en la modernidad. Del monoteismo religioso al politeismo cultural, Barcelona, Anthropos.

BILBAO, A. (1999), "La administración burocrática crea las condiciones de la confianza”, en R. Ramos Torre y F. García Selgas (dir.), Globalización, riesgo, reflexividad, Madrid, CIS, pp. 311-330.

BOURDIEU, P. (1998), La distinción, Madrid, Taurus.

BOYER, R. (ed.) (1986), La flexibilidad del trabajo en Europa, Madrid, Ministerio de Trabajo y Seguridad Social.

CALLEJO, J. (1996), "Fatalidad del mercado y culturas de la producción”, Sociología del trabajo, $\mathrm{n}^{\circ} 26$, invierno $1995 / 1996$, pp. 29-62.

(2001), "Crisis de la encefalopatía espongiforme: huecos en el modelo social de consumo", Estudios sobre consumo, $\mathrm{n}^{\circ} 58, \mathrm{pp} .47-60$.

DAKE, K. y A. WILDAVSKY (1990), "Theories of risk perception: who fears what and why?", Daedalus, monográfico sobre riesgo, 119, Vol. (4), pp. 41-60.

DOUGLAS, M. (1992), Risk and Blame, Londres, Routledge.

(1996), La aceptabilidad del riesgo según las ciencias sociales, Barcelona, Paidós.

(1998), Estilos de pensar, Barcelona, Gedisa.

FISCHLER, C. (1995), El (h)omnivoro, Barcelona, Anagrama.

GALBRAITH, J. K. (1984 [1958]), La sociedad opulenta, Barcelona, Planeta-Agostini.

GIDDENS, A. (1995), Modernidad e identidad del yo, Barcelona, Península.

(1997), "Vivir en una sociedad postradicional", en U. Beck, A. Giddens y S. Lash (eds.), Modernización reflexiva, Madrid, Alianza.

GIDDENS, A., Z. BAUMAN, N. LUHMANN y U. BECK (1996), Las consecuencias perversas de la modernidad, Barcelona, Anthropos.

GIL CALVO, E. (2003), El miedo es el mensaje. Riesgo, incertidumbre y medios de comunicación, Madrid, Alianza Editorial.

JAKUS, P.M. y W.D. SHAW (2003), "Perceived Hazard and Product Choice: An Application to Recreational Site Choice", The Journal of Risk and Uncertainty, vol. 26 (1), pp. 77-92.

LASH, S., B. SZERSZINSKY, y T. WYNNE, (eds.), Risk, Environment and Modernity: Towards a New Ecology, Londres, Sage. 
LUHMANN, N. (1992), Sociología del riesgo, Guadalajara (Mex.), Universidad Iberoamericana y Universidad de Guadalajara.

(1998), Sistemas sociales, Barcelona, Anthropos.

MARX, K. y F. ENGELS (2002), El manifiesto comunista, Barcelona, Crítica.

MILLÁN, A. (2002), "Malo para comer, bueno para pensar. Crisis en la cadena socioalimentaria", en M. Gracia Arnaiz (coord), Somos lo que comemos, Barcelona, Ariel.

NEIRA GONZÁLEZ, M. (2003), "En qué dirección va la seguridad alimentaria”, Revista Española de Salud Pública, $\mathrm{n}^{\circ}$ 77, pp. 307-311.

OFFE, C. (1990), Contradicciones en el Estado del Bienestar, Madrid, Alianza Editorial.

RAMOS, R. (1999), "Prometeo y las flores del mal: el problema del riesgo en la sociología contemporánea", en R. Ramos Torre y F. García Sélgas (dir.), Globalización, riesgo, reflexividad, Madrid, CIS.

RITZER, G. (1999 [1993]), La McDonalización de la sociedad. Un análisis de la racionalización en la vida cotidiana, Barcelona, Editorial Ariel.

RITZER, G. y A. LISKA, (1997), "McDisneyization and post-tourism. Complementary perspectives on contermporary tourism", en C. Rojek y J. Urry (eds), Transformation of travel and theory. Touring cultures, Londres, Routledge.

RITZER, G. y S. MILES, (1998), "McDonalization and the Global Sports Store: Constructing Consumer Meanings in a Rationalized Society", en M. Alfino, J. S. Caputo y R. Wynyard (eds.), McDonalization Revisited. Cultural Essays on Consumer Culture, Westpor, Praeger.

SEN, A. (1982), Choice, Welfare and Measurement, Cambridge (Mass.) y Londres, Harvard University Press.

SILVERSTONE, (2004), ¿Por qué estudiar los medios?, Buenos Aires, Amorrortu.

VISCUSI, V.K. y W.N. EVANS (1998), "Estimation of Revealed Probabilites and Utility Functions for Product Safety Decisions", Review of Economics and Statistics, vol. 80, pp. 28-33.

WEBER, M. (1979), Economia y Sociedad, México, Fondo de Cultura Económica.

ZUCKER, l. (1986), "Production of trust: institutional sources of economic structure, 1840-1920", en Shaw, B.M. y L. L. Cummings (eds.), Research in Organizational Begavior, Vol. 8, Greenwich, JAI-Press. 